\title{
Crystallographic Orientation Maps Obtained from Ion and Backscattered Electron Channeling Contrast
}

\author{
C. Lafond ${ }^{1}$, T. Douillard ${ }^{1}$, S. Cazottes ${ }^{1}$, S. Dubail ${ }^{2}$ and C. Langlois ${ }^{1}$ \\ 1. MATEIS laboratory - INSA Lyon, Lyon, France. \\ 2. Axon Square Ltd, Sciez, France.
}

For several years now, new directions have been explored to obtain orientation maps by other means than the classical EBSD setup or to modify it in order to get improved information. Particularly, the channeling contrast may be used to obtain orientation map. In few words, the channeling contrast consists in a variation of the secondary and backscattered electron yields when the orientation of a crystal is changed compared to the beam position, or when defects are present just under the sample surface. To use the channeling contrast for orientation mapping, the main concept of our work is to acquire an electron or ion image series when rotating a pre-inclined polycrystalline sample with respect to the beam. Along such image series, each $(\mathrm{X}, \mathrm{Y})$ pixel of the region of interest undergoes an intensity variation that can be plotted as a function of the rotation angle. Such intensity profiles can be theoretical predicted for a given orientation of a crystal, using a "particle" approach when ion beam is used, or a "diffraction" approach when using backscattered electrons. The indexation procedure then relies on a search in a database of theoretical profiles obtained by simulating intensity profiles for a large set of orientation matrices sampling statistically the SO3 orientation space. In the following, the acronym iCHORD will stand for ion CHanneling Orientation Determination, and eCHORD for the same method but using electrons.

Concerning the iCHORD method, the proof of concept was obtained on a TiN sample [1] and successfully tested on other cubic materials $(\mathrm{Cu}, \mathrm{Fe}, \mathrm{Co}, \mathrm{Ni})$. In order to get insight on the angular resolution of the iCHORD method, EBSD measurements on the same area could be used. However, distortions between the electron scanning electronic and the ion scanning electronic prevent a direct comparison of the orientation pixel-to-pixel. To evaluate the angular resolution in a self-consistent manner, one can acquire a first image series, and another one after rotating the sample of few degrees, on the same area. The experiment was carried out on a nickel sample and two iCHORD maps were acquired with a rotation of $6^{\circ}$ of the sample before acquiring the second map. When plotting the distribution of disorientation values between the two iCHORD maps, a Gaussian distribution is found, centered on 6.4 degrees, but also with a root mean square value of around 0.6 degree (see Figure1). This value has to be compared with the angular precision of the database itself. In our example, the database used for indexing contained 500000 theoretical profiles, with a precision of 0.63 degree. A better result would be possibly obtained with a lager database (i.e. better angular precision) but at the expense of computation time. Even with a larger database, the angular resolution would reach a limit value because of issues related to the alignment of the images, precession during the rotation, planeity of the sample. The value of 0.6 degree is therefore a representative value of the angular resolution of the iCHORD method.

Concerning the eCHORD method, the initial problem was the simulation of the experimental profiles. Thanks to the similarity between the eCHORD method and Electron Channeling Patterns (ECP) acquisition, it appears that the experimental intensity profiles can be effectively simulated by extracting the intensity along a circle from simulated ECPs, calculated using the codes from $\mathrm{M}$. De Graef and co- 
workers [2]. A database of theoretical profile was then constructed, used for indexing the experimental profiles acquired on a nickel sample (see Figure 2). The role of the initial tilt angle, the high tension used, as well as the energy distribution of the BSE used to construct the ECPs in the M. De Graef codes are critical for the quality of the simulation and will be discussed.

As a conclusion, the angular resolution of approximately $0.6^{\circ}$ for the iCHORD method may be sufficient for some application of orientation maps, in a compromise between angular resolution, spatial resolution, indexation speed and even 3D capabilities (under study). For orientation maps obtained from an electron image series, the main advantage is that no extra detector is needed to carry out the experiment, opening orientation mapping capability potentially on any SEM machine. The geometry of acquisition, simpler than the EBSD one, could be also a critical advantage when turning to 3D orientation mapping.

References:

[1] C. Langlois et al, Ultramicroscopy 157 (2015), p. 65

[2] P.G. Callahan and M. De Graef, Microscopy and Microanalysis 19 (2013), p. 1255

a)

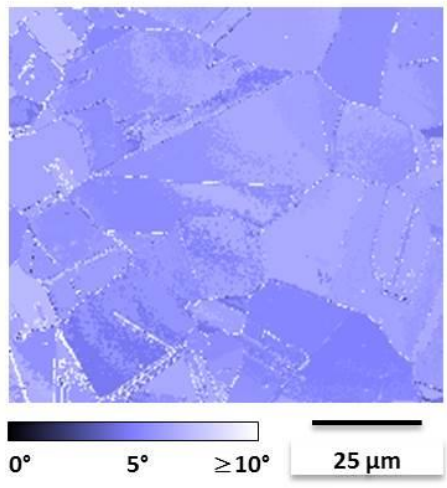

b)

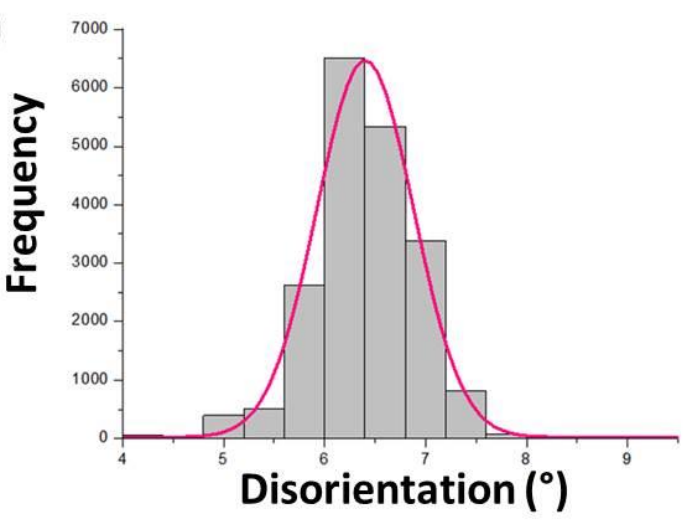

Figure 1. (a) Disorientation map between two iCHORD maps acquired with an orientation delta of $6^{\circ}$ applied to the sample. (b) Distribution of disorientation values fitted with a Gaussian distribution centered on 6.4 , with a rms value of $0.6^{\circ}$.
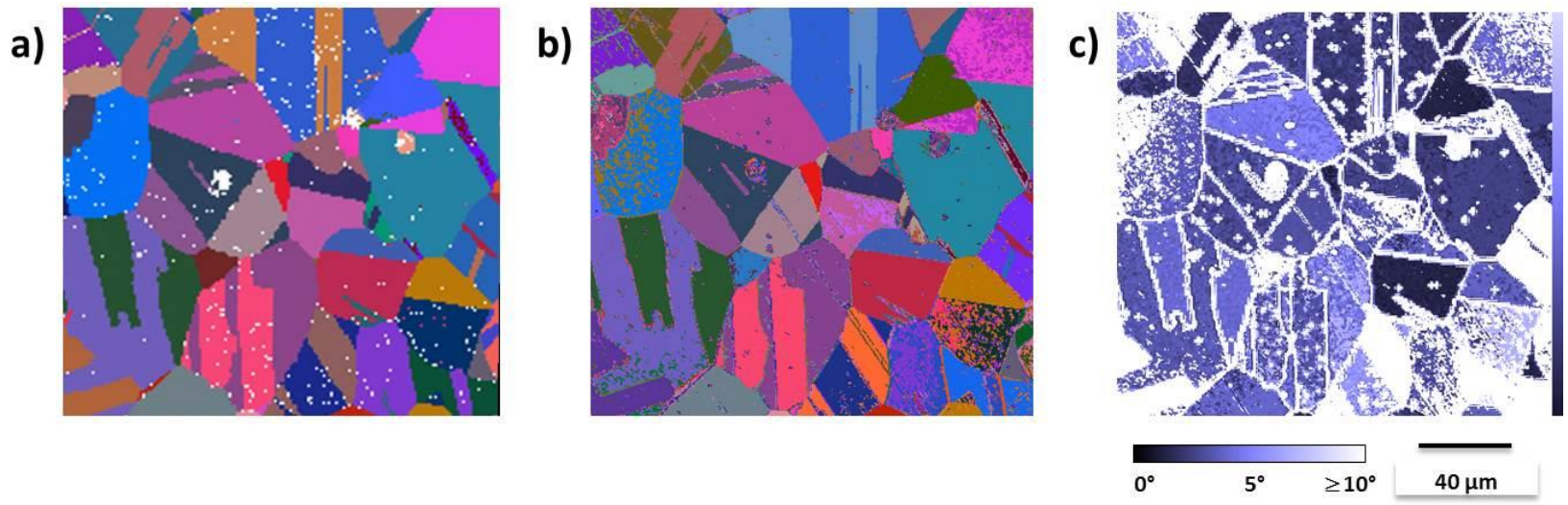

Figure 2. (a) EBSD (b) eCHORD map (c) disorientation map between eCHORD and EBSD maps. 